\title{
Effect of Nv Shi Solid Drink on Endocrine Regulation in Mice with Kidney-Yin Deficiency Induced by Levothyroxine Sodium
}

\author{
Shuo Liu ${ }^{1}$, Si-yao Ma ${ }^{1}$, Wenyi Zhu ${ }^{2}$, Jia Liang ${ }^{3}$, Yu-jing Shi ${ }^{4}$, Hong Meng ${ }^{2 *}$ \\ ${ }^{1}$ Research and Development of Healthy Food, Infinitus (China) Co., Ltd., Guangzhou, China \\ ${ }^{2}$ Cosmetics Science and Technology, Beijing Technology and Business University, Beijing, China \\ ${ }^{3}$ Traditional Chinese Medicine, Graduate School of Chinese Academy of Traditional Chinese Medicine, Beijing, China \\ ${ }^{4}$ Institute of Chinese Materia Medica, China Academy of Chinese Medical Sciences, Beijing, China
}

\author{
*Corresponding author: Hong Meng, \\ Cosmetics Science and Technology, Beijing \\ Technology and Business University, No. \\ 11/33, Fucheng Road, Haidian District, \\ Beijing, China \\ Tel.: +861061374229 \\ Email: menghong2000@163.com
}

Received May 09, 2020

Revised May 26, 2020

Accepted June 10, 2020

Published June 30, 2020

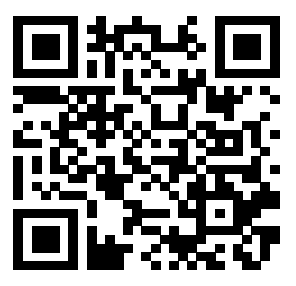

\begin{abstract}
Purpose: To observe the effects of Nv Shi solid drink on thyroid hormone, sex hormones and cyclic nucleotide of kidney-yin deficiency hyperthyroid mice induced by levothyroxine sodium. Methods: ICR mice were randomly grouped into normal control group, model group, Nv Shi solid drink high dose group, the middle dose group and low dose group, male and female in half. The kidney-yin deficiency mice models were established by intragastric administration of levothyroxine sodium before intragastric administration of corresponding drugs once a day for 10 consecutive days. One day after the last administration, the effects were observed by measuring weight. And tetraiodothyronine (T4), triiodothyronine (T3), thyrotropin (TSH), testosterone (T), estradiol (E2), cyclic adenosine monophosphate (cAMP), cyclic guanosine monophosphate (cGMP) were tested by ELISA method. Results: Compared with the model group, the body weight increased significantly $(p<0.05)$, T3 level decreased significantly in high and medium dose group $(p<0.01, p<0.05)$, and serum T4 and cAMP decreased significantly $(p<0.01, p<0.05)$, TSH increased significantly $(p<0.01, p<0.05)$ in all dose groups, and cAMP/cGMP decreased in the low dose group $(p<0.05)$. The expression of E2 in female decreased significantly in all does groups, the same as the ratio of $E 2 / T$ in high dose group $(p<0.05)$. There was no significant change in the expression of sex hormones in male in all dose groups $(p>0.05)$. Conclusion: Nv Shi solid drink may improve the kidney-yin deficiency syndrome by adjusting T3, T4, TSH, cAMP and E2 level in the endocrine system of the body and exert the function of nourishing yin and tonifying kidney.
\end{abstract}

Keywords: Nv Shi solid drink, Kidney-yin deficiency, Sex hormone, Thyroid hormone, Cyclic nucleotide

\section{Introduction}

肾阴虚证是指由于肾阴亏损, 失于滋养, 虚热内生所表现的 证候（Wang \& Tian, 2008)。目前有多种肾阴虚证动物模型用 于功效评价 (Lin et al., 2013), 其中甲状腺机能六进肾阴 虚证模型较为常用 (Wang, 2013)。这种方法是通过给实验动 物使用甲状腺素, 形成外源性甲状腺素水平升高, 造成实验动 物甲状腺机能六进, 促进体内物质代谢, 增加耗氧量, 使产热 增加, 能够模拟甲状腺功能六进时的消瘦、怕热、多汗、神经 过敏、急躁、震颤、心率加快、心输出量增加等症状, 表现基
本符合临床肾阴虚的辨证诊治（Zhang et al., 2015; Fu et al., 2017)。

女仕牌固体饮料由黑木耳、获苓、枸杞子、桑椹、龙眼肉、 玉竹、薏蔌仁、莲子、大東等药食同源的中药组成, 具有滋肾 阴、补阴血的功效。本实验采用甲状腺激素法建立肾阴虚证动 物模型, 探讨女仕牌固体饮料对肾阴虚动物模型的影响并阐释 作用机制, 为阐明药食同源中药复方品种滋阴补肾功效的作用 机制提供实验依据。 


\section{Methods}

\section{Instruments and materials}

1) Test instrument

全自动多功能酶标仪 (MULTISKAN MK3; 美国Thermo公司, 美 国）；电热恒温培养箱（DH4000A；天津泰斯特公司，中国） ; 低温离心机 (5810R；德国Eppendorf公司，德国）；MINI shaker (MH-1; Kylin-Bell Lab Instruments QILINBEIER公 司，中国）。

\section{2) Material}

女仕牌固体饮料（批号 $18 \mathrm{E} 31 \mathrm{EAC01}$; 无限极 (中国)有限公司, 中国); 左甲状腺素钠 (批号248922, Merk Serono GmbH, 德 国）；Mouse E2 elisa kit（CSB-E05109m，武汉华美生物工程 有限公司, 中国); Mouse T elisa kit（CSB-E05101m, 武汉 华美生物工程有限公司, 中国）; Mouse T3 elisa kit（CSBE05109m，武汉华美生物工程有限公司，中国）; Mouse T4 elisa kit（CSB-E05083m; 武汉华美生物工程有限公司, 中国）; Mouse TSH elisa kit (CSB-E05116m; 武汉华美生物工程有限公 司，中国）; Mouse cAMP elisa kit（20181024; 南京建成生物 工程研究所, 中国) ; Mouse cGMP elisa kit（20181024; 南京 建成生物工程研究所, 中国）。

\section{3) Animal}

ICR小鼠 (SPF级), 雌雄兼用, 体重为 18-20 g, 购自北京华 阜康生物科技股份有限公司, 动物合格证号： 11401300086884 , 使用许可证号：SCXK（京）2015-0041。

\section{Experimental method}

1) Animal grouping and treatment

将ICR小鼠分为 5 组, 每组 20 只, 雌雄各半。正常组、模型组、 女仕牌固体饮料大 $(1.65 \mathrm{~g} / \mathrm{kg} / \mathrm{d})$ 、中 $(0.83 \mathrm{~g} / \mathrm{kg} / \mathrm{d})$ 、小剂量组 $(0.41 \mathrm{~g} / \mathrm{kg} / \mathrm{d})$, 分别相当于成人日用量的 2 倍, 1倍, 0.5 倍。 除正常组外, 各组小鼠给予左甲状腺素钠 $50 \mu \mathrm{g} / \mathrm{kg} \quad \mathrm{ig}, 0.1$ $\mathrm{mL} / 10 \mathrm{~g}$, 每天 1 次, 连续 10 天, 造成肾阴虚证候小鼠模型。 每日给予左甲状腺素钠后间隔 2 小时给予相应药物 $0.2 \mathrm{~mL} / 10$ $\mathrm{g}$ ig, 连续 10 天, 正常组和模型组在同等条件下给予蒸馏水。

\section{2) Detection of index}

每天观察大鼠饮食饮水情况、精神状态、毛色、体态, 末次 给药后称取体质量。观察小鼠一般体征。第 11 天称重后眼眶 取血, 离心分离血清, $-20^{\circ} \mathrm{C}$ 保存待测, 采用ELISA方法检测甲状 腺素（thyroxine，T4）、三碘甲状原氨酸（triiodothyronine, T3）、促甲状腺激素（thyroid-stimulating hormone, TSH）、 睪酮（testosterone，T）、雌二醇（estradiol， E2）、环磷酸 腺苷 (cyclic Adenosine monophosphate, cAMP) 、环磷酸鸟苷 (cyclic guanosine monophosphate, cGMP) 的表达。

\section{Statistical analysis}

各组所得数据均以（mean士standard deviation）表示，组间 比较采用单因素方差分析。

\section{Results}

\section{Effect of Nv Shi solid drink on general physical signs in mice} with kidney-yin deficiency

正常组小鼠毛发润泽、活动自如; 连续给予左甲状腺素钠 10 天后, 模型组小鼠出现躁动、易惊、体毛粗䊅无光泽等表现, 体重增长缓慢, 与正常组比较有极显著性差异 $(p<0.01)$ 。灌 胃给予女仕牌固体饮料后小鼠毛色和光泽度有所改善, 易惊表 现有改善趋势。女仕牌固体饮料大剂量、中剂量组小鼠体重增 加, 与模型组比较均有显著性差异 $(p<0.05)$ 。见Table 1 。

2. Effect of Nv Shi solid drink on hyperthyroidism in mice with kidney-yin deficiency

给予左甲状腺素钠后, 模型组小鼠血清TSH含量降低, T3、 $\mathrm{T} 4$ 含量升高, 与正常组比较均有显著性差异 $(p<0.01)$; 女仕 牌固体饮料各剂量组血清TSH含量升高, 与模型组比较有显著 性差异（ $p<0.01 ， p<0.05 ）$; 女仕牌固体饮料大剂量、中剂量 组血清T3含量均降低, 与模型组比较有显著性差异 $(p<0.01, p$ $<0.05 ）$ ；女仕牌固体饮料各剂量组血清T4含量降低，与模型组

Table 1. Effect of Nv Shi solid drink on body mass in mice with kidney-yin deficiency

\begin{tabular}{lcc}
\hline Group & Dosage $(\mathrm{g} / \mathrm{kg} / \mathrm{d})$ & After model establishment $(\mathrm{g})$ \\
Normal group & - & $28.50 \pm 1.27^{1)}$ \\
Model group & - & $24.80 \pm 1.40^{\# \#}$ \\
High dose group & 1.65 & $26.70 \pm 2.11^{*}$ \\
Middle dose group & 0.83 & $26.60 \pm 2.17^{*}$ \\
Low dose group & 0.41 & $25.89 \pm 2.80$ \\
\hline
\end{tabular}

${ }^{1)}$ Mean \pm standard deviaiton; Compared with normal group, ${ }^{\# \#} p<0.01$; Compared with model group, ${ }^{*} p<0.05$. 
比较有极显著性差异 $(p<0.01)$ 。见Table 2。

\section{Effect of Nv Shi solid drink on the level of sex hormone in mice with kidney-yin deficiency}

对于雌性小鼠：模型组血清E2含量升高, T含量降低, E2/T升 高, 与正常组比较均有显著性差异 $(p<0.01, p<0.05)$; 给予女 仕牌固体饮料后, 各剂量组雌鼠 $\mathrm{E} 2$ 水平降低, 大剂量组雌鼠 $\mathrm{E} 2 / \mathrm{T}$ 水平降低, 与模型组比较均有显著性差异 $(p<0.05)$ 。

对于雄性小鼠: 模型组血清T含量降低, E2/T升高, 与正常组 比较均有显著性差异 $(p<0.05)$, 模型组血清E2含量无显著改 变, 与正常组比较均无显著性差异 $(p>0.05)$; 给予女仕牌固体 饮料后, 大剂量和小剂量组雄鼠血清E2/T有降低趋势, 但与模型 组比较无显著性差异 $(p>0.05)$ 。见Table 3 。

\section{Effect of Nv Shi solid drink on cyclic nucleotide level in mice with kidney-yin deficiency}

模型组小鼠血清cAMP含量升高, cAMP/cGMP升高, 与正常组比 较均有显著性差异 $(p<0.05)$, cGMP无显著变化 $(p>0.05)$; 给 予女仕牌固体饮料后, 各剂量组小鼠血清cAMP含量降低, 与模
型组比较均有显著性差异（ $p<0.05 ）$; 女仕牌固体饮料各剂量 组CGMP含量, 与模型组比较均无显著差异 $(p>0.05)$; 女仕牌固 体饮料小剂量组 $c A M P / c G M P$ 降低, 与模型组比较均有显著性差异 $(p<0.05)$ 。见Table 4 。

\section{Discussion}

肾阴虚是中医学虚证的基本证型之一, 是指由于肾阴亏损, 失于滋养, 虚热内生所表现的证候, 肾阴虚证见腰膝酸痛, 失 眠多梦, 头晕耳鸣, 咽干颧红, 五心烦热, 遗精早泄, 潮热 盗汗, 舌红少津无苔, 脉细数等 (Fu et al., 2017)。临床肾 阴虚患者多见体形消瘦、皮肤干燥、心烦易怒, 模型组小鼠体 重减轻一定程度上说明虚损的程度。肾阴虚主要与甲状腺轴、 性腺轴、能量代谢、免疫力下降等有关 ( Fu et al., 2017; Dai et al., 2016; Zou et al., 2015)。中医认为, 甲状腺 机能六进属阴虚证范畴 (Huang et al., 2008), T3、T4升高多 见于五心烦热, 咽干口燥, 舌红少苔, 脉细数的肾阴虚型患 者 (Fang, 2008)。甲状腺功能六进可出现血清 T3、T4含量明

Table 2. Effect of Nv Shi solid drink on serum TSH, T3, T4 level in in mice with kidney-yin deficiency

$(\mathbf{N}=\mathbf{2 0})$

\begin{tabular}{llll}
\hline Group & TSH $(\mathrm{ulU} / \mathrm{mL})$ & $\mathrm{T} 3(\mathrm{ng} / \mathrm{mL})$ & $\mathrm{T} 4(\mathrm{ng} / \mathrm{mL})$ \\
Normal group & $6.29 \pm 1.61^{1)}$ & $4.02 \pm 0.87$ & $227.40 \pm 68.55$ \\
Model group & $1.59 \pm 0.29^{\# \#}$ & $5.45 \pm 0.93^{\# \#}$ & $594.05 \pm 91.15^{\# \#}$ \\
High dose group & $2.53 \pm 0.67^{* *}$ & $4.11 \pm 0.88^{* *}$ & $379.72 \pm 62.88^{* *}$ \\
Middle dose group & $2.16 \pm 0.65^{*}$ & $4.38 \pm 0.67^{*}$ & $422.98 \pm 65.65^{* *}$ \\
Low dose group & $2.14 \pm 0.76^{*}$ & $4.78 \pm 0.79$ & $442.44 \pm 78.91^{* *}$ \\
\hline
\end{tabular}

${ }^{1}$ Mean \pm standard deviaiton; TSH, thyroid-stimulating hormone; T3, triiodothyronine; T4, thyroxine; Compared with normal group, ${ }^{\# \#} p<0.01$; Compared with model group, ${ }^{*} p<0.05,{ }^{* *} p<0.01$.

Table 3. Effect of Nv Shi solid drink on serum E2, T level and ratio of E2/T in mice with kidney-yin deficiency

$(\mathrm{N}=\mathbf{2 0})$

\begin{tabular}{lllllcc}
\hline \multirow{2}{*}{ Group } & \multicolumn{2}{c}{ TSH $(\mathrm{ulU} / \mathrm{mL})$} & \multicolumn{2}{c}{$\mathrm{T3}(\mathrm{ng} / \mathrm{mL})$} & \multicolumn{2}{c}{$\mathrm{T} 4(\mathrm{ng} / \mathrm{mL})$} \\
\cline { 2 - 7 } Normal group & \multicolumn{1}{c}{ Female } & Male & Female & Male & Female & Male \\
Model group & $184.57 \pm 9.31^{1)}$ & $170.33 \pm 19.49$ & $0.84 \pm 0.09$ & $2.75 \pm 0.35$ & $221.47 \pm 30.72$ & $63.10 \pm 13.82$ \\
High dose group & $191.04 \pm 23.06^{*}$ & $165.44 \pm 38.67$ & $0.74 \pm 0.10$ & $1.73 \pm 0.15$ & $261.75 \pm 32.96^{*}$ & $95.96 \pm 22.44$ \\
Middle dose group & $190.26 \pm 26.28^{*}$ & $178.11 \pm 26.48$ & $0.62 \pm 0.10$ & $1.69 \pm 0.24$ & $221.47 \pm 30.72$ & $63.10 \pm 13.82$ \\
Low dose group & $188.72 \pm 15.91^{*}$ & $0.65 \pm 0.06$ & $0.84 \pm 0.09$ & $2.75 \pm 0.35$ & $334.50 \pm 60.83^{\# \#}$ & $102.55 \pm 33.18^{\#}$ \\
\hline
\end{tabular}

${ }^{1)}$ Mean \pm standard deviaiton; TSH, thyroid-stimulating hormone; T3, triiodothyronine; T4, thyroxine; Compared with normal group, ${ }^{\#} p<0.05,{ }^{\# \#} p<0.01$; Compared with model group, ${ }^{*} p<0.05$.

Table 4. Effect of Nv Shi solid drink on serum cAMP and cGMP level and the ratio of cAMP/cGMP in mice with kidney-yin deficiency (N=20)

\begin{tabular}{llll}
\hline \multicolumn{1}{c}{ Group } & cAMP $(\mathrm{nmol} / \mathrm{L})$ & cGMP $(\mathrm{nmol} / \mathrm{L})$ & $159.62 \pm 24.77$ \\
Normal group & $139.52 \pm 22.28^{1)}$ & $143.35 \pm 28.36$ & $0.89 \pm 0.18$ \\
Model group & $160.45 \pm 15.42^{\#}$ & $1.16 \pm 0.25^{\#}$ & $1.02 \pm 0.19$ \\
High dose group & $145.46 \pm 15.01^{*}$ & 149.5821 .43 & $1.00 \pm 0.22$ \\
Middle dose group & $143.38 \pm 21.80^{*}$ & $154.93 \pm 12.60$ & $0.94 \pm 0.09^{*}$ \\
Low dose group & $145.84 \pm 13.09^{*}$ & 1.86 \\
\hline
\end{tabular}

${ }^{11}$ Mean \pm standard deviaiton; Compared with normal group, ${ }^{\#} p<0.05$; Compared with model group, ${ }^{*} p<0.05$. 
显升高, 基础代谢增强 (Huang et al., 2011), 负反馈抑制腺 垂体分泌TSH，二者互相拮抗，T3、T4、TSH表达异常能反映 甲状腺轴功能紊乱 (Dai et al., 2016)。在临床检查中, 性激 素E2、T和 $E 2 / T$ 比值异常能反映性腺轴功能紊乱, 是阴阳失调 的重要指标 (Xu et al., 2007)。但各类肾虚类型的T与E2水平 及变化不尽相同 (Qiu et al., 1999)。有研究将E2含量上升、 $\mathrm{T}$ 含量下降, $\mathrm{E} 2 / \mathrm{T}$ 作为肾阴虚大鼠模型造模成功的评判标准 (Peng \& Zhang., 2003)。环核苷酸cAMP、cGMP是调节细胞功能 活动的重要物质, 二者相互制约、相互拮抗, 其对生物细胞的 双向调节作用与中医阴阳学说相似 (Cheng, 2016; Li et al., 2017 )。cAMP/cGMP升高可反映肾阴虚动物模型造模成功 (Luo, 2006)。本实验中, 左甲状腺素钠诱导肾阴虚模型小鼠出现躁 动、易惊、体毛粗粘无光泽, 体重增长缓慢等状态, 表现虚损 症状。肾阴虚小鼠血清T3、T4含量上升, TSH含量下降, T含量 下降、E2/T比值上升, cAMP/cGMP比值升高, 均表明肾阴虚模 型造模成功。

女仕牌固体饮料由黑木耳、获苓、枸杞子、桑椹、龙眼肉、 玉竹、薏药仁、莲子、大杳等药食同源的中药组成, 其中以黑 木耳、枸杞子、桑椹为君, 可滋阴补血、补肝益肾、生津止 渴, 缓解头军眼花、潮热盗汗; 获苓、龙眼肉、莲子为臣, 可 宁心安神, 清心除烦、缓解失眠心烦、汗出; 玉竹、薏药仁为 佐, 可健脾益气、养阴润燥, 缓解口干口燥; 大冭为使, 可补 气养血, 调和口味, 全方共奏滋肾阴、补阴血的功效。本研究 采用左甲状腺素钠诱导肾阴虚模型评价女仕牌固体饮料干预肾 阴虚的作用, 分为低、中、高剂量组, 在造模后给予女仕牌固 体饮料干预, 不仅观察女仕牌固体饮料干预肾阴虚的功效, 同 时初步探讨对肾阴虚的作用机制。

机体处于甲方状态与下丘脑-垂体-甲状腺轴（hypothalamicpituitary-thyroid, HPT) 相关 (Duan et al., 2016), 甲元 型肾阴虚小鼠的甲状腺功能六进、基础代谢增多, 躁动、易惊 扰, 体毛干枯、体重下降, 血清T3, T4 含量升高, TSH含量降 低。本实验结果显示, 女仕牌固体饮料可改善肾阴虚模型动物 毛色和光泽度, 在一定程度上改善模型动物的易惊状态, 并可 增加模型动物体重, 具有降低肾阴虚动物模型血清 $T 3 、 T 4 、$ 升 高TSH作用。研究从分子水平提示, 女仕牌固体饮料能改善甲 状腺功能六进, 改善HPT轴功能。

中医认为 “肾藏精, 主生长发育与生殖”, 肾气的盛衰直接 影响着人体的生殖功能, 而现代医学也发现生殖系统的功能受 下丘脑-垂体-性腺（hypothalamic-pituitary-gonadal axis, $H P G$ ) 轴神经内分泌调节, 肾阴虚与HPG轴功能密切相关 (Zhang et a1., 2017)。本实验结果显示女仕牌固体饮料可降低雌性 肾阴虚动物模型血清E2含量, 降低E2/T比值, 研究提示女仕牌 固体饮料能在一定程度上调节性激素平衡, 改善 $\mathrm{HPG}$ 轴功能。

机体处于正常状态下, 血清的 cAMP, cGMP水平不会发生变
化, 一旦机体持续处于亢奋状态, 通过激活腺苷酸环化酶的 活性, 诱导 $\mathrm{cAMP}$, cGMP的合成。cAMP和cGMP是调节物质代谢和 生物学功能的重要物质, 对细胞功能处于稳定状态具有双向控 制调节作用 (Pan et al., 2014), 且与中医学的阴阳学说有相 似之处, 研究表明, cAMP含量升高是肾阴虚的特征之一(Chen, 2011), 本实验结果显示女仕牌固体饮料可降低肾阴虚动物模 型血清CAMP含量, 降低CAMP/cGMP比值, 研究提示女仕牌固体 饮料在一定程度上具有平衡环核苷酸系统的作用。

\section{Conclusion}

女仕牌固体饮料能在一定程度上改善肾阴虚小鼠虚损状态, 调节肾阴虚证的相关物质基础促甲状腺激素（TSH）、三碘甲 状腺原氨酸 (T3) 、甲状腺素 (T4) 、环磷酸腺苷 (cAMP) 以 及雌性小鼠䧳二醇 (E2) 水平, 从而改善甲状腺功能六进, 调 节雌性肾阴虚模型性激素, 平衡环核苷酸系统。研究提示, 女 仕牌固体饮料可能通过改善肾阴虚动物模型神经内分泌HPT轴 和HPG轴功能, 平衡环核苷酸系统, 从而发挥滋阴补肾功效。

\section{Author's contribution}

SL, YJS and HM designed the experiment and YJS, WYZ and JL operated the animal experiment. SL and SYM analyzed the data and YJS and HM wrote the article.

\section{Author details}

Shuo Liu (Engineer), Research and Development of Healthy Food, Infinitus (China) Co., Ltd., Hongtai Wisdom Valley, 19th of Si Cheng Rd, Tian He District, Guangzhou 510663, China; Si-yao Ma(Engineer), Research and Development of Healthy Food, Infinitus (China) Co., Ltd., Hongtai Wisdom Valley, 19th of Si Cheng Rd, Tian He District, Guangzhou 510663, China; Wen-yi Zhu (Graduate student), Cosmetics Science and Technology, Beijing Technology and Business University, 11 Fucheng Road, Haidian District, Beijing 10048, China; Jia Liang (Graduate student), Traditional Chinese Medicine, Graduate School of Chinese Academy of Traditional Chinese Medicine, 16 Dongzhimen neinan street, Dongcheng District, Beijing 100700, China; Yu-jing Shi (Associate Research Fellow), Institute of Chinese Materia Medica, China Academy of Chinese Medical Sciences, Yinghua street, Chaoyang District, Beijing 110105, China; Hong Meng (Professor), Cosmetics Science and Technology, Beijing Technology and 
Business University, 11 Fucheng Road, Haidian District, Beijing 10048, China.

\section{References}

Cheng SK. The combination of traditional Chinese and western medicine and Its Enlightenment (3)- Professor Kuang Ankun opened up a "ice breaking trip" for the study of "Yin Yang theory" of traditional Chinese Medicine. Chinese Journal of Integrated Traditional and Western Medicine, 36: 12851289, 2016.

Chen Q. Research methods in pharmacology of Chinese materia medica. People's Medical Publishing House, Beijing, p1028, 2011.

Duan SJ, Chen JF, Tan W, Liang J, Yan YY. Correlation between adrenal cortical function and kidney deficiency syndrome in autoimmune thyroid disease. Chinese Journal of Integrative Medicine on Cardio-/Cerebrovascuiar Disease, 14: 2227-2229, 2016.

Dai B, Yang ML, Zhang JN, Li YX, Ouyang LQ, Xiao ZZ, Deng $J M$, Yang L. Effect of Liuweidihuang decoction and "Yibu Yixie" on HPT Axis in kidney-yin deficiency mice model. Information on Traditional Chinese Medicine, 33: 19-22, 2016.

Fang ZQ. Syndrome differentiation and treatment of traditional Chinese medicine. Shanghai University of Traditional Chinese Medicine Press, Shanghai, p566, 2008.

Fu HE, Li JM, Liu YH. Effect of Zuoguiwan on neuro-endocrineimmune function of kidney-yin deficiency rats. Chinese Journal of Experimental Traditional Medical Formulae, 23: 155-159, 2017.

Huang DB, Hu XG, Pu GX. Pharmacology and clinic of Integrated Chinese and Western Medicine. Hubei Science \& Technology Press, Wuhan, p434, 2008.

Huang JR, Li XH, Zhang JJ, Du YM, Zhang Y. Effect of Liuwei Dihuang Wan on Shenyi vacuity model mice with hyperthyroidism by thyroxin and reserpine. Pharmacology and Clinics of Chinese Materia Medica, 27: 1-3, 2011.

Li YJ, Wei DD, Wei ML, Wang XK, Zhang Y, Yue XX, Ding PN, Yang XY, Cui LH. Evaluation of three kinds of kidney yin deficiency modeling based on animal substance and energy metabolism detection. Chinese Journal of Chinese Medicine, 32: 83-88, 2017.

Lin ZJ, Zhang B, Liu XQ, Zhu WJ. Research on animal model evaluation with traditional Chinese medicine principles. China Journal of Traditional Chinese Medicine and Pharmacy, 28: 2217-2221, 2013.

Pan X, Hu CJ, Geng YY, Zhao L, Xiong R, Chen ZM. T lymphocyte sub-population and CAMP/cGMP regulating effect by processed ershen pill formula. Chinese Journal of Experimental Traditional Medical Formulae, 13: 138$141,2014$.

Peng Y, Zhang YD. Research status of kidney-deficiency and sex hormone. Heilongjiang Journal of Traditional Chinese Medicine, 6: 50-51, 2003.

Qiu RX, Wu GZ, Jin MH. Regulating effect of sex hormone on the balance of patients with kidney-yin and kidneyyang deficiency. Chinese Journal of Basic Medicine in Traditional Chinese Medicine, 3: 46-47, 1999.

Wang D, Tian FH. Research on kidney-yin deficiency. Journal of Liaoning University of TCM, 10: 33-35, 2008.

Wang P, Wang XJ. Research overview of animal model with kidney-yin deficiency. Information on Traditional Chinese Medicine, 30: 123-125, 2013.

Zhang ZY, Chen BJ, Zhang YJ, Wang WT, Wang YR, Su YX. Establishment and stability observation of rat model with kidney-yang deficiency and kidney-yin deficiency. Fujian Journal of TCM, 46: 51-54, 2015.

Xu XQ, Huang JY, Luo R, Xiao BQ, Zhao T, Tu XY. Effect of active fraction in erjing pill on learning dysmnesia of rat with kidney-yin deficiency and its molecular mechanism. Chinese Traditional and Herbal Drugs, 38: 564-569, 2007.

Zhang JN, Li YX, Yang ML, Xiao ZZ, Dai B, Xiao WZ, Ouyang LQ. Effects of Liuwei Dihuang decoction and "reinforcing and reducing" herb couples on HPG axis in kidney Yin deficiency mice. Chinese Journal of Modern Applied Pharmacy, 34: 25-29, 2017.

Zou HM, Zhang B, Sun W, Zhao ப, Yang YT, Zhao AH, Qiu MF. Research progress in biochemical indicators of syndrome of deficiency of kidney Yin. China Journal of Traditional Chinese Medicine and Pharmacy, 30: 3607-3610, 2015. 


\section{中文摘要}

\section{女仕牌固体饮料对左甲状腺素钠诱导肾阴虚小鼠模型内分泌系统的影响}

刘硕 ${ }^{1}$, 马思遥 ${ }^{1}$, 朱文驿 ${ }^{2}$, 梁佳 $^{3}$, 时宇静 ${ }^{4}$, 孟宏 $^{*}$

${ }^{1}$ 无限极（中国）有限公司健康食品研发，广州，中国

${ }^{2}$ 北京工商大学化妆品科学与技术, 北京, 中国

${ }^{3}$ 中国中医科学院研究生院中医学, 北京, 中国

${ }^{4}$ 中国中医科学院中药研究所, 北京, 中国

目的: 观察女仕牌固体饮料对左甲状腺素钠致肾阴虚模型小鼠体内甲状腺激素、性激素及环核苷酸的影响。方 法: ICR小鼠随机分为正常组、模型组、女仕牌固体饮料大、中、小剂量组, 雌雄各半, 采用左甲状腺素钠片灌 胃造成小鼠肾阴虚模型, 造模当天灌胃给予样品, 每天1次, 连续10天。末次给药后1天, 观察小鼠体征及体 重, 取血, 采用Elisa方法检测血清中甲状腺素（thyroxine, T4）、三碘甲状原氨酸（triiodothyronine, T3）、 促甲状腺激素（thyroid-stimulating hormone, TSH）、睪酮（testosterone, T)、雌二醇（estradiol, E2）、 环磷酸腺苷 (cyclic Adenosine monophosphate, CAMP) 、环磷酸鸟苷 (cyclic guanosine monophosphate, cGMP) 的表达。结果：与模型组比较，女仕牌固体饮料大剂量组和中剂量组小鼠体重显著升高 $(p<0.05)$; 大 剂量和中剂量组T3显著降低 $(p<0.01, p<0.05)$ ，各剂量组血清T4、CAMP均显著降低 $(p<0.01, p<0.05)$, TSH均显著升高 $(p<0.01, p<0.05)$ ，小剂量组cAMP/ cGMP比值降低 $(p<0.05)$, 各剂量组雌鼠E2表达显著 降低, 大剂量组雌鼠E2/T比值降低, 各剂量组雄鼠性激素表达无显著改变（ $p>0.05 ）$ 。结论: 女仕牌固体饮料 可能通过调整机体内神经分泌系统中T3、T4、TSH、CAMP、雌鼠E2这些共同的潜在物质基础, 改善HPT轴和 HPG轴功能、平衡环核苷酸系统，从而改善肾阴虚证，发挥泫阴补肾功效。

关键词: 女仕牌固体饮料，肾阴虚证，性激素，甲状腺激素，环核苷酸 


\title{
국문초록
}

\section{Levothyroxine Sodium에 의해 유발 된 신장음질 결핍 마우스의 내분비 조절에 대한 여성브랜드 고형 음료의 효능}

\author{
류석 ${ }^{1}$, 마사요 ${ }^{1}$, 주문이 ${ }^{2}$, 양가 $^{3}$, 시우정 ${ }^{4}$ 맹홍 ${ }^{2 *}$ \\ ${ }^{1}$ 무한급(중국)유한회사 건강식품개발, 광주, 중국 \\ ${ }^{2}$ 북경공상대학 화장품과학과 기술, 북경, 중국 \\ 3중국중의과학원연구생원 중의학, 북경, 중국 \\ 4중국중의과학원중약연구소, 북경, 중국
}

목적: Levothyroxine sodium에 의해 유발 된 신장 음질 결핍 마우스에서 갑상선 호르몬, 성 호르몬 및 사이 클릭 뉴클레오티드에 대 한 여성 브랜드 고형 음료의 효능을 확인하고자 한다. 방법: ICR 마우스를 정상 대조군, 모델 군, 여성브랜드 고형 음료 고용량 군, 중간 용량 군 및 저용량 군, 수컷 및 암컷으로 무작위로 그룹화 하였다. 신장음질 결핍 마우스 모델은 10 일 연속으로 하루에 한 번 levothyroxine sodium을 위내 투여에 의해 형성되었다. 마지막 투여 1 일 후, 체중을 측정함으로써 그 효과를 관찰 하였다. ELUSA 법으로 혈청 내 thyroxine (T4) triiodothyronine, (T3), thyroid-stimulating hormone (TSH), testosterone (T), estradiol (E2), cyclic adenosine monophosphate (cAMP), cyclic guanosine monophosphate (cGMP)의 발현을 측정하였다. 결과: 모델 그룹과 비 교하여, 여성브랜드 고형 음료의 고용량 및 중간 용량 그룹에서 마우스의 체중은 유의하게 증가하였고( $p<0.05)$; 고용량 및 중간 복 용량 그룹에서 T3는 유의하게 감소 하였다 $(p<0.01, p<0.05)$. 혈청 T4 및 $\mathrm{CAMP}$ 는 유의하게 감소하였고 $(p<0.01, p<0.05)$, TSH는 유 의적으로 증가하였고 $(p<0.01, p<0.05)$, 저용량 그룹 $\mathrm{cAMP} / \mathrm{cGMP}$ 비율은 감소 하였다 $(p<0.05)$, 각 용량 그룹 암컷 쥐에서 $\mathrm{E} 2$ 의 발 현은 유의하게 감소하였고 고용량 군에서 암컷 쥐의 $\mathrm{E} 2 / \mathrm{T}$ 비율은 감소하였으나, 각 용량 군에서 수컷 쥐에서 성 호르몬의 발현에는 유의 한 변화가 없었다( $p>0.05)$. 결론: 여성브랜드 고형 음료는 신체의 내분비 계에서 T3, T4, TSH, cAMP 및 E2 수준을 조절하여 신장 음질 결핍 증후군을 개선할 수 있다.

핵심어: 여성 브랜드 고형 음료, 신음 결핍 증후군, 성 호르몬, 갑상선 호르몬, 사이클릭 뉴클레오티드 
